\title{
Poincaré Partially Integrable Local Representations and Mass-Spectrum
}

\author{
Moshé Flato and Daniel Sternheimer \\ Physique Mathématique, Collège de France, Paris - France
}

Received January 17, 1969

\begin{abstract}
An example is given of an irreducible representation of a finitedimensional Lie algebra containing the Poincaré Lie algebra and giving rise to isolated positive masses. In addition the representation is Poincare partially integrable (which assures the continuous physical spectrum for the energy-momentum vector) and "Poincaré-covariant" in a weak sense.

A connection between this example and some recently published impossibility theorems is shown, and conclusions about a possible future work in this domain are also drawn.
\end{abstract}

\section{Introductory Remarks and Definitions}

Some time ago an example was given [1] of an irreducible representation of a finite-dimensional Lie algebra containing a Poincaré subalgebra for which the squared mass operator is self-adjoint and admits only isolated masses. This example, valid as a counterexample to a no-masssplitting theorem conjectured earlier [2], suffered from obvious difficulties as to its physical interpretation. In particular the example of [1], which is partially integrable on its translation subalgebra [contrarily of the affirmation of [3]: it is just that the domain of integrability of the translation subalgebra does not coincide with the invariant domain of the entire $\mathfrak{s u}(2,2)$ Lie algebra; in that example the energy-momentum $P_{\mu}$ is taken on its maximal domain of definition], is not integrable on the Lorentz part of $\mathfrak{s u}(2,2)$. Would it be Poincaré-partially-integrable [4] then according to [5] the spectrum of the energy-momentum vector $P_{\mu}$ would have to be continuous. The fact that the irreducible representations of the translation group are all one-dimensional shows that there is no surprise in the fact that in [1] the spectrum of $P_{\mu}$ admits gaps of the order of magnitude of the mass-splitting (a fact which was also mentioned by [3]).

In [3] a corollary of a rigorous no-go theorem (applied to finite dimensional Lie groups and proved by Jost and SEgAL [6]) is proved which makes it possible to apply the result of [6] to a very restricted family of non-integrable (or local) representations of Lie algebras. It is only the fact that one could think (as it is claimed in [3]) that practically 
no interesting example of mass splitting can be found within the framework of non-integrable representations of finite-dimensional Lie algebras containing Poincaré, that urges us to present a new, Poincaré partially integrable, example.

Moreover, in [3], it is left unclear in what sense does the result generalize the theorem of Jost and SEgAL. One immediate remark will be that if together with the finite translation invariance of [3] one also supposes (a thing which is more than reasonable since the common domain of [3] is also the "mass-spectrum domain") that the domain contains one analytic vector for one element of the Lie algebra, then the domain will necessarily contain (by the assumed finite translation invariance) many common analytic vectors for a whole set of elements of the Lie algebra. Therefore the hypotheses of [3] are quite near to the integrability of a direct component containing Poincaré, for which we already have the theorem [6]. The example which we shall show later hints also at the fact how integrability is connected with translational invariance.

An interesting different approach to our problem - which however demands another and stronger type of hypotheses - was recently discussed by Coleman and Mandula [7]. These authors try to gain an additional information about the coupling between space-time and internal symmetries from the $n$-particle states. Indeed, assuming that the group giving rise to mass-spectrum is a symmetry-group of a non-trivial $S$-matrix when acting in the generally admitted way on the $n$-particle states, and modulo very strong assumptions of "particle-finiteness", analyticity, etc., a direct sum coupling is proved for a large family of groups and representations. One immediately remarks that in [7] the group hypothesis is quite crucial [otherwise one meets difficulties with the existence of a nice invariant common dense domain and with finite Poincare invariance for the Lie algebra; it seems that for non-integrable representations the theorem of [7] will not hold generally], and that the result of [7] should by no means be applied to spectral-unifications (sometimes refered to as "spectrum-generating-algebras" or "dynamicalgroups", or "non-invariance-groups") since by definition they are not symmetry groups of $S$-matrix.

Before we pass to our example we make the following remarks:

a) By a representation of a finite dimensional Lie algebra, we understand a mapping of the algebra to the ring of operators acting on Hilbert space (each operator with its own domain) such that there exists a common invariant dense domain on which the operators satisfy the commutation relations of the Lie algebra. Physical operators (and their spectral decomposition) will therefore be considered on other (larger) domains than the common invariant dense domain. 
b) By irreducibility of a Lie algebra representation we understand Schur-irreducibility, namely that every bounded operator commuting with all the representing-operators is a multiple of the identity operator.

To be precise, we shall say that the bounded operator $B$ commutes with the representing-operator $X$ if $B X \varphi=X B \varphi$ for all $\varphi$ in some dense subspace (obviously contained in the domain of $X$ ). We could have given stronger definitions and especially, if $X$ is (e.g.) skew-adjoint and if we write $B=B_{1}+i B_{2}$, with $B_{1}$ and $B_{2}$ self-adjoint, require that the spectral resolutions of $B_{1}$ and $B_{2}$ commute with that of $X$. However, if we have Schur-irreducibility with the former (weak) definition of commutation, it is evident that we shall have also commutation according to all stronger definitions, and therefore all definitions of Schur-irreducibility are equivalent. It will therefore be enough for us, in the following (and as far as Schur-irreducibility is concerned), to suppose commutation in the above weak (and more practical) sense.

c) One can define a domain of partial integrability (as we do) as a domain which contains a dense set of common analytic vectors for all elements of the given subalgebra. In such a case it is evident (in virtue of [3]) that no dense domain can be found in our Hilbert space which will be invariant under the entire Lie-algebra, be of Poincaré-partialintegrability, and give rise to a non-connected mass-spectrum. In accordance with remark a), we shall need at least two different domains (as indeed will be seen later from our example): one for partial integrability and mass-spectrum, the other being the common invariant dense domain for the whole Lie algebra.

Would one define a domain of partial integrability in a non-constructive way, namely by the existence of a unique connected group of operators, the infinitesimal generators of which close to the given subalgebra on the given domain, then it is an open question (and we believe a difficult one) if one can find a finite-dimensional algebra of operators with a unique dense domain invariant under the entire Lie algebra, which is at the same time a domain of Poincaré-partial-integrability in this sense and contains eigenvectors corresponding to different isolated masseigenvalues.

d) What is the possible physical interpretation of the two domains? The first domain, which is the one for Poincare partial integrability and mass spectrum, has a very clear role: it is the domain on which all generators of Poincare are represented by operators having the right spectral properties for every particle, and thus describing the kinematics of every particle and the mass-spectrum of all particles.

The second domain which is the common invariant dense domain under the whole algebra (in our example it is contained in the first domain) has quite a different role to play: it is the domain on which 
the representation of the entire algebra is irreducible, namely on which all particles (characterized as mass eigenvectors) are glued together.

A one-particle state which belongs of course to the first domain can in our example be expressed as an infinite series of vectors belonging to the second domain.

\section{The Example}

These remarks being made we pass to the example itself: Let $D_{J}^{+}\left(m_{0}\right)$ be a unitary irreducible representation of the universal covering $\widetilde{P}$ of the Poincaré group $P$ corresponding to a mass $m_{0}>0$, spin $J$ and positive energy. The representation space $H_{1}$ will be realized in the usual way:

$$
H_{1}=\sum L_{j}^{2}\left(R^{3} ; \frac{d^{3} p}{p_{0}}\right)(j=-J, \ldots,+J)
$$

Denote by $M_{\mu \nu}^{\prime}$ and $P_{\mu}^{\prime \prime}$ the generators of the Lie algebra $\mathscr{P}$ of $P$ in our representation. Now let $H$ be the Hilbert space $L^{2}(0, a) \hat{\otimes} H_{1}$ : evidently a vector in $H$ is a function of $p$, a discrete variable $j$ (taking $2 J+1$ values, as in [5]), and of $q \in(0, a)$. Now we put $M_{\mu \nu}=I \bar{\otimes} M_{\mu \nu}^{\prime}$ and $P_{\mu}^{\prime}=I \bar{\otimes} P_{\mu}^{\prime \prime}$ (where $I$ stands for the identity operator on $L^{2}(0, a)$ and the bar stands for the closure). In this obvious manner does $\mathscr{P}$ act on $H$. For the sake of clarity we notice that $P_{\mu}^{\prime \prime}$ and $M_{\mu \nu}^{\prime}$, being the infinitesimal generators of a unitary representation of $\widetilde{P}$ on $H_{1}$, have their usual domains of definition on $H_{1}$, and therefore so do $M_{\mu \nu}$ and $P_{\mu}^{\prime}$ on $H$.

Next we define $i \partial_{q}$ on a domain with periodic boundary conditions. The operators $P_{\mu}=-i P_{\mu}^{\prime} \partial_{q}$ will be skew-adjoint (on a domain of vectors which are $C^{\infty}$ periodic in $q$ and have in the $p$ variables the same behaviour as the domain of $P_{\mu}^{\prime}$ in $H_{1}$ ) and we shall make use of them as well as of the operator $A=i q$ defined in $H$.

Let $S_{\pi}$ be the domain in $H$ of functions which are $C^{\infty}$ with rapid decrease in the momentum variables $p$ and $C^{\infty}$ periodic in $q$. Let $S_{0}$ be the subspace of $S_{\pi}$ of functions vanishing (with all their derivatives) for $q=0$ and $q=a$. Evidently both domains are dense in $H$. The introduction of $S_{0}$ is imposed upon us as $S_{\pi}$ is not left invariant under the action of $A=i q$, while $S_{0}$ does, as can be trivially remarked. $\left(S_{\pi}\right.$ and $S_{0}$ will play the role of the two needed domains as explained before).

On $S_{0}$ one can therefore check the relations $\left[P_{\mu}, A\right]=P_{\mu}^{\prime}$. On the other hand, the relations $\left[P_{\mu}^{\prime}, A\right]=0=\left[M_{\mu \nu}, A\right]$ can be checked both on $S_{0}$ an $S_{\pi}$. Generally speaking one will be able to check on $S_{\pi}$ the commutation relations of the Lie algebra

$$
\mathscr{P}_{2}=\mathscr{L} \cdot\left(T_{4} \oplus T_{4}^{\prime}\right) \supset \mathscr{L} \cdot T_{4} \approx \mathscr{P}
$$


where $\mathscr{L}$ is generated by the $M_{\mu \nu}, T_{4}$ by the $P_{\mu}$ and $T_{4}^{\prime}$ by the $P_{\mu}^{\prime}$. All these operators are essentially skew-adjoint on $S_{\pi}$ and the semi-direct product is defined by the representation $D(1 / 2,1 / 2) \oplus D(1 / 2,1 / 2)$ of $\mathscr{L}$.

We have therefore here a representation $T(G)$ of the Lie algebra $G \approx A_{0} \cdot \mathscr{P}_{2}=\left(\mathscr{L} \oplus A_{0}\right) \cdot\left(T_{4} \oplus T_{4}^{\prime}\right)$, where $A_{0}$ is the one-dimensional Lie algebra generated by $A$ acting on $T_{4} \oplus T_{4}^{\prime}$ by $\left(\begin{array}{ll}0 & 0 \\ -I_{4} & 0\end{array}\right)$.

It can be trivially checked that this representation is Schur-irreducible (as stated before this is the kind of irreducibility in which we are interested - our representation is even topologically irreducible, in the sense it is a "closure" of an algebraically irreducible one), with the dense common invariant domain $S_{0}$.

Moreover it is partially integrable to the group $\widetilde{P}_{2}=S L(2, C) . R^{8}$ (the Lie algebra of which is $\mathscr{P}_{2}$ ) on the domain $S_{\pi}$.

If we identify the physical Poincare group $\widetilde{P}$ with the one the Lie algebra of which is generated by the $M_{\mu \nu}$ and $P_{\mu}$, we find by reduction on $\widetilde{P}: \sum_{n=1}^{\infty} D_{J}^{+}\left(n m_{0}^{\prime}\right) \oplus D_{J}(0) \oplus \sum_{n=1}^{\infty} D_{J}^{-}\left(n m_{0}^{\prime}\right)$, where $m_{0}^{\prime}=\frac{2 \pi m_{0}}{a}$, $D_{J}^{ \pm}(m)$ stands for the representation of $\widetilde{P}$ with mass $m$, spin $J$, and positive (or negative) energy, and $D_{J}(0)$ for the non-faithful representation of $\widetilde{P}$ obtained from the previous for vanishing energy-momentum $P_{\mu}$.

We have thus the mass relation $m=n m_{0}^{\prime}$, where $m_{0}^{\prime}$ is our basic mass and $n$ is a positive integer. We shall interpret the $D_{J}(0)$ as a vacuumtype representation [though as one can notice our vacuum is infinitely degenerate - contrarily to what is usually assumed in field-theory - a natural feature of such kind of models; however the corresponding vacuum subspace is cyclic in our representation space], the $D_{J}^{+}$as a representation corresponding to real physical particles and the $D_{\bar{J}}$ as unphysical representations (or alternatively as antiparticles, using the hole theory of Dirac). One may remark that if one insists on getting rid after reduction of the $D_{J}^{-}$representations which correspond to negative energies, this is also possible if one complicates enough the model: As a matter of fact one can look at the 19-dimensional Lie algebra generated by $T(G)$ (which is our 15-dimensional former represented algebra) and the four generators $Q_{\mu}=\left(-\partial_{q}^{2} P_{\mu}^{\prime}\right)$ on a domain with vanishing boundary conditions for $\partial_{q}^{2}$, and of course one has to replace $S_{\pi}$ by the space $S_{e}$ of $C^{\infty}$ functions, rapidely decreasing in $p$, and vanishing for $q=0$ and $q=a$ together with their even-order derivatives in $q$. Thus if we identify in this model the physical Poincare Lie algebra as the one generated by the $M_{\mu \nu}$ and the $Q_{\mu}$ (on $S_{e}$ ), we get the reduction $\sum_{n=1}^{\infty} D_{J}^{+}\left(n^{2} m_{0}^{\prime \prime}\right)$, where 
$m_{0}^{\prime \prime}=\left(\frac{\pi}{a}\right)^{2} \cdot m_{0}$, of the obtained representation of the physical Poincaré group into its irreducible components.

\section{Poincaré Covariance and Other Remarks}

We now remark (restricting ourselves to our first example) that the use of the Poincaré-group will have its full meaning only if we succeed in incorporating the Poincaré covariance to our scheme. We already know the representatives $T(X)$, where $X \in G$, are antisymmetric operators on the common invariant domain $S_{0}$. Let $U=(\exp T)(a, \tilde{\Lambda})$ be the unitary representative of the element $(a, \widetilde{\Lambda}) \in \widetilde{P}$. Now the operators $U T(X) U^{*}$ satisfy on $U S_{0}$ the same commutation relations that satisfy the $T(X)$ on $S_{0}$. Moreover we have $U S_{\pi}=S_{\pi}$ (and contains $S_{0}$ and $U S_{0}$ ), which assures the fact that physical observables (like rest masses, etc. . .) will not depend on a particular choice of a Lorentz frame. We have thus established the Poincaré-covariance of our representation, though in a somehow weaker sense than that postulated in field-theory. $(T(G)$ and $S_{0}$ are in this interpretation respectively the representation and the common invariant dense domain corresponding to no-motion for the "all particles'picture"). This weak form of Poincaré-covariance translates in a simple mathematical language the principles of special theory of relativity. We therefore think that it suffices for physical applications. In any case if we permit the existence of two different Poincaré groups, the spectral one (the Lie algebra of which is generated by the $M_{\mu \nu}$ and $P_{\mu}=-i P_{\mu}^{\prime} \partial_{q}$ ) and the covariance one (the Lie algebra of which is generated by the same $M_{\mu \nu}$ and by the $P_{\mu}^{\prime}$ ), in such a case we can establish the Poincaré covariance of our example (here covariance stands in the usual sense of field-theory) even on $S_{0}$.

We now make the following remarks concerning the degree of arbitrariness and the physical character of our example.

1. From what was said before it is clear that we have no ambiguity as to the non-unique extension of operators from $S_{0}$ to $S_{\pi}$ etc. All our operators are first defined on $S_{\pi}$ (with exception of $A=i q$ which is defined everywhere) and then we look at their restriction to the domain $S_{0} \subset S_{\pi}$ - an operation which is uniquely defined.

2. It is true that one could have chosen instead of $S_{\pi}$ the domain $S_{\pi}^{(t)}$ which is defined from $S_{\pi}$ by replacing (in the Fourier series in $q$ ) $n$ by $n+t(0<t<1)$, giving rise to the mass relation $m=m_{0}^{\prime}(n+t)$. Besides of mentioning that this will be just another representation according to our definitions, we give three reasons why to prefer our representation making use of $S_{\pi}$ to the other possible ones:

a) If we want to have the possibility of defining a generalized vacuumstate (from the kind stated before) we have to have the eigenvalue $P_{\mu}=0$ in our spectrum. This already forces the choice of $t=0$.

21 Commun.math. Phys., Vol.12 
b) Only the choices of $t=0$ and $t=1 / 2$ will give a symmetrical spectrum for $P_{0}$, a thing which is desired if we want to have for every eigenvalue of $P^{2}$ at least one real particle.

c) Among the spaces $S_{\pi}^{(t)}$, we know that $S_{\pi}^{(0)}=S_{\pi}$ is a one which singles out: it is only $S_{\pi}$ which is spanned by a complete set of singlevalued functions on the circle in $q$, and for many evident reasons the coordinate $q$ will have the advantage in being considered as a circular coordinate.

\section{Conclusions}

We are now in position of establishing the connection between our example and the impossibility theorems discussed at the beginning. As far as the theorem of Jost and SEgat is concerned, there is no problem. We just do not have an integrable representation, i. e. we cannot pass to a representation of a corresponding Lie group. However our example is partially integrable on $S_{\pi}$ on the 14-dimensional Lie algebra $\mathscr{P}_{2}$ : it is only $A_{0}$ which prevents $T(G)$ from being integrable on $S_{\pi}$; in this sense our example is an optimal one.

As to the result of [3], our example shows that the hypotheses under which it was proved are indeed too restrictive: if we only permit the domain of partial integrability (namely the mass-spectrum domain) to be different from the common invariant domain of the Lie algebra representation, the result of [3] cannot be applied.

As to the theorem of [7] two remarks are made: first is that our example shows quite clearly how the group assumption, existence of common invariant domain which is also invariant under finite Poincaré unitary transformations, etc., are needed for the proof, which is therefore simply not valid local representations. Secondly - for the case of group representations - it seems to be interesting in connection with [7] to clarify what basic differences exist (if at all) between the usual Poincaré invariant $S$-matrix and the a priori possible non-trivial $S$-matrix invariant under our $\widetilde{P}_{2}$ group on $H$.

Moreover it seems to us that situations of the kind described by the $\widetilde{P}_{2}$ group were not taken into account in [7]: as a matter of fact it was quite explicitely supposed in [7] that the state-labelling of particles will consist of the Poincaré labelling in momentum space plus additional indices not related with momentum space - a thing which is not satisfied in the case of the $\widetilde{P}_{2}$ group on $H$.

We now conclude our article with the following three remarks:

1. It is possible to continue this promising direction in the following ways: 
a) The construction of a non-trivial nicely behaving $S$-matrix admitting $\widetilde{P}_{2}$ as its invariance group (or admitting the 15-dimensional Lie algebra $G$ as its invariance algebra).

b) The study of more complicated examples (as hinted by the example of the 19-dimensional algebra) e. g. with spin spectrum, and of other types of local representations.

c) Introduction of internal symmetries (like $S U(3)$ ) to such schemes by similar techniques.

2. Looking for "physically acceptable" mass-spectrum, people have been interested in the last years in the so-called infinite-component field theories. Though there exists a connection between this technique and the general problem of Lie algebras representation, we think that as far as the greatest success of such theories lies in the existence of a nicely behaved mass (and spin) spectrum, we prefer to utilize our technique which does not bring any new doubtful field concept to the theory, and thus escaping from the well-known field-theoretical diseases of the infinite component theories.

3. People have been trying last years to find a non-trivial relativistically invariant representation of current-algebra at infinite momentum. It might be well pointed out that besides of the formal resemblance between this last problem and our example - both being the search for Lie algebras representations - there exists another connection between them: in fact once the Lie algebra representation is given, an invariant operator can be constructed and utilized as a formal Lagrangian of the theory. Then one can calculate the algebra of currents from this Lagrangian (which in some simple cases is isomorphic to the original algebra). The original representation space is then utilized to saturate the current-algebra. Of course in such cases one should have an infinite spin spectrum - but such examples are immediately derived by means of our technique.

\title{
References
}

1. Flato, M., and D. Sternhemmer: Phys. Rev. Letters 16, 1185 (1966).

2. O'Raifeartaigh, L.: Phys. Rev. Letters 14, 575 (1965).

3. - Phys. Rev. 164, 2000 (1967).

4. Doebner, H. D., and O. Melsueimer: Nuovo Cimento 49, 73 (1967).

5. Wigner, E.: Ann. Math. 40, 149 (1939).

6. Jost, R.: Helv. Phys. Acta 39, 369 (1966).

SEgAL, I. E.: J. Funct. Anal. 1, 1 (1967).

7. Coleman, S., and J. Mandula: Phys. Rev. 159, 1251 (1967).

\author{
M. FLATO \\ Institut Henri Poincaré \\ 11, rue P.-M. Curie \\ 75 Paris $\left(5^{e}\right)$, France
}

